\title{
The current status of suicide and self-injury in eating disorders: a narrative review
}

\author{
Katrina Kostro ${ }^{1 *}$, Jessica B Lerman ${ }^{2}$ and Evelyn Attia ${ }^{1,2}$
}

\begin{abstract}
The aim of this paper is to review recent literature on suicide and self-injury in eating disorders (ED) including anorexia nervosa (AN), bulimia nervosa (BN), and binge eating disorder (BED). Among psychiatric diagnoses, EDs are associated with increased mortality rates, even when specialized treatment is available. Of the mortalities that are reported in individuals with EDs, suicide is among the most commonly reported causes of death. Additionally, suicidal and non-suicidal self-injurious behaviors occur frequently in this clinical population. A literature search was undertaken using the databases of Medline/PubMed and Psyclnfo to identify papers describing suicidality in individuals with ED diagnoses. The authors identified studies and review articles published between 2005-2013 (inclusive) that describe the relationship between EDs and suicide, and associated behaviors including self-injurious behaviors, or non-suicidal self-injury (NSSI). The initial search resulted in 1095 papers that met the a priori search criteria. After careful review, 66 papers were included. The majority of papers described clinical cohorts that were studied longitudinally. The diagnosis described most frequently in selected studies was AN. There are limited current data about the prevalence of suicide and NSSI among individuals with EDs. Among the published studies that focus specifically on the relationship between EDs and suicidality, most describe AN in more detail than other EDs. Nonetheless, rates of mortality, and specifically rates of suicide, are undeniably high in ED populations, as are the rates of self-harm. Therefore, it is critical for clinicians and caretakers to carefully evaluate these patients for suicide risk and to refer promptly for appropriate treatment.
\end{abstract}

Keywords: Anorexia nervosa, Bulimia nervosa, Binge eating disorder, Eating disorders, Suicidality, Suicide, Mortality, Self-injury, Non-suicidal self-injury, Review

\section{Background}

Eating disorders (ED), including anorexia nervosa (AN) and bulimia nervosa $(\mathrm{BN})$, are serious psychiatric illnesses with high rates of morbidity and mortality. Studies of clinical samples, mostly from specialized programs in Europe and North America, have described high rates of suicidal behaviors and completed suicides associated with EDs, and have helped increase clinical awareness about the seriousness of these psychiatric disorders [1-6]. A comprehensive review published in 2006 by Franko and Keel describes findings from 1985-2004 [7]. This review highlighted findings of high suicide rates among individuals with $\mathrm{AN}$, and contrasted these with individuals with $\mathrm{BN}$, whose rates of suicide did not appear to be elevated. Conversely, the review reported that non-lethal suicide attempts occurred in $25-35 \%$ of $\mathrm{BN}$ patients, compared with a rate of $3-20 \%$ of

\footnotetext{
* Correspondence: katrinakostro@gmail.com

'Columbia University Medical Center, New York, NY, USA

Full list of author information is available at the end of the article
}

AN patients [7]. The review also described common clinical correlates of suicidality in EDs, including comorbid diagnoses such as depression, self-injurious behaviors such as bingeing and purging, a history of substance abuse, and a history of childhood abuse.

Considering the consistent finding of high risk of suicidality in this population, it is important for researchers and clinicians to continue to examine the risk factors and associations with the prevalence of suicidality in individuals with EDs. This narrative review aims to update the previous review by Franko and Keel [7], and to extend the scope to include non-suicidal self-injury (NSSI). The authors included NSSI in this review as these behaviors are commonly reported in EDs and were not included in many earlier reports of suicidality in this clinical population. The authors aimed to include publications that described all ED diagnosis groups, including binge eating disorder (BED) and eating disorder not otherwise specified (EDNOS), as many earlier reviews focused primarily on $\mathrm{AN}$ and $\mathrm{BN}$. The 
authors aimed to describe the available data, including their quality and scope and to identify areas in need of additional study.

\section{Review \\ Methods}

The authors performed a literature search between July and December 2013, using the databases of Medline/PubMed and PsycInfo, to review papers published between January 1, 2005 and December 31, 2013, describing suicidality and self-injury in individuals with ED diagnoses. The start date for the search interval was selected because the most recently published comprehensive review on similar subject matter described studies published between 1985-2004. Twenty advanced searches were undertaken, combining ED key words with suicidality key words. The first and second key words of each search were required to appear in the article title or abstract. The ED key words used were: "eating disorders"; "anorexia nervosa"; "bulimia nervosa"; "binge eating disorder"; or "eating disorder not otherwise specified". Each of the five ED terms was paired with a second key word of the following key words: "suicide"; "mortality"; "self-injurious behavior"; or "non-suicidal self-injury". Additionally, the authors conducted four searches, combining the ED key words "avoidant/restrictive food intake disorder" and "ARFID" with each of the four selected second key words to identify any recent literature that may have been published in anticipation or soon following the release of DSM-5 in May 2013.

Articles were included in this review if studies included information about the relationship between suicidality or self-injury and individuals with ED diagnoses including AN, BN, BED, EDNOS and ARFID. Articles were excluded if they did not present data about suicidality or self-injury in EDs, or if they did not identify and discuss a specific relationship between EDs and suicidality or self-injury. Only articles in English were reviewed. The articles were reviewed by all authors of this paper.

\section{Results}

\section{Quality appraisal of included papers}

The literature search of suicidality and EDs produced overlapping lists of published studies and review articles. The initial search resulted in 1095 papers that met the a priori search criteria (Figure 1). Seventy-four duplicate articles were removed, as were 69 articles written in languages other than English, and 886 papers were excluded due to failure to include data regarding suicidality in EDs. The authors ultimately identified 66 papers that met the inclusion criteria and present them in Table 1 including information about study type, sample size, age group and gender. The literature search results are highlighted in narrative text organized by ED diagnosis and then by general features relevant to risk of suicidality and EDs.
The literature included variation regarding methodology, sample size, length of follow-up, and diagnostic criteria that may account for the variability among studies. Overall, the authors found that data on the prevalence of suicidality in EDs have focused mainly on AN. Interpretation of the more limited data about $\mathrm{BN}$ is difficult because information regarding initial ED diagnosis is rarely available and diagnostic cross-over from AN to $\mathrm{BN}$ is high, with estimated cross-over rates ranging from 20-50\% [10]. Additionally, many studies examined predictors of poor outcome in EDs but did not specify predictors of mortality. Furthermore, the cause of patient deaths, including death by suicide, was not always readily available, and thus limited the ability to interpret study findings. Also, there were few studies assessing the cause of mortality in patients with BN, BED, and other EDs not otherwise specified.

\section{Anorexia nervosa}

Until recently, the majority of research regarding increased mortality risk conferred by EDs, including risk of suicidality, has focused on AN. Across ED diagnoses, recent studies examining the characteristics of suicide attempts among individuals with $\mathrm{AN}, \mathrm{BN}$, and individuals without EDs, found higher Standardized Mortality Ratios (SMR) in AN than in $\mathrm{BN}$, ranging from 6.2 to $10.6[34,39,52]$. SMR represents the observed number of deaths divided by the expected number of deaths in a certain group, and thus represents the estimated risk of death in a group compared to individuals from the general population, matched by age and gender. A clinical cohort study published by Bulik et al. in 2008 reported suicide attempts approaching approximately $17 \%$ in the AN population [14]. According to Guillaume et al., the odds ratio (OR) for suicide attempts in AN compared to other ED diagnoses showed that AN patients were more likely to have made a serious attempt (OR 3.4, 95\% CI 1.4-7.9) [34]. The results also showed that the AN group with suicide attempts had a higher expectation of dying (OR 3.7, 95\% CI 1.1-13.5) based on scores from The Suicidal Intent Scale, a 15-item scale that assesses the severity of suicidal intention associated with suicide attempts [34]. Huas et al. concluded that in AN patients with a lifetime history of suicide attempt, risk of death was multiplied by 2.6 [38].

Reported rates of suicide attempts in AN are high, and there are also data confirming completed suicides in individuals with AN. Huas et al. reported that suicide was the second highest cause of death $(17 \%, n=7)$ in a clinical cohort with AN, $28.4 \%$ of which had committed at least one suicide attempt [38]. Papadopoulos et al. found that the SMR of observed deaths caused by suicide in a study population defined by having been admitted at least once for treatment of $\mathrm{AN}$, compared to expected 


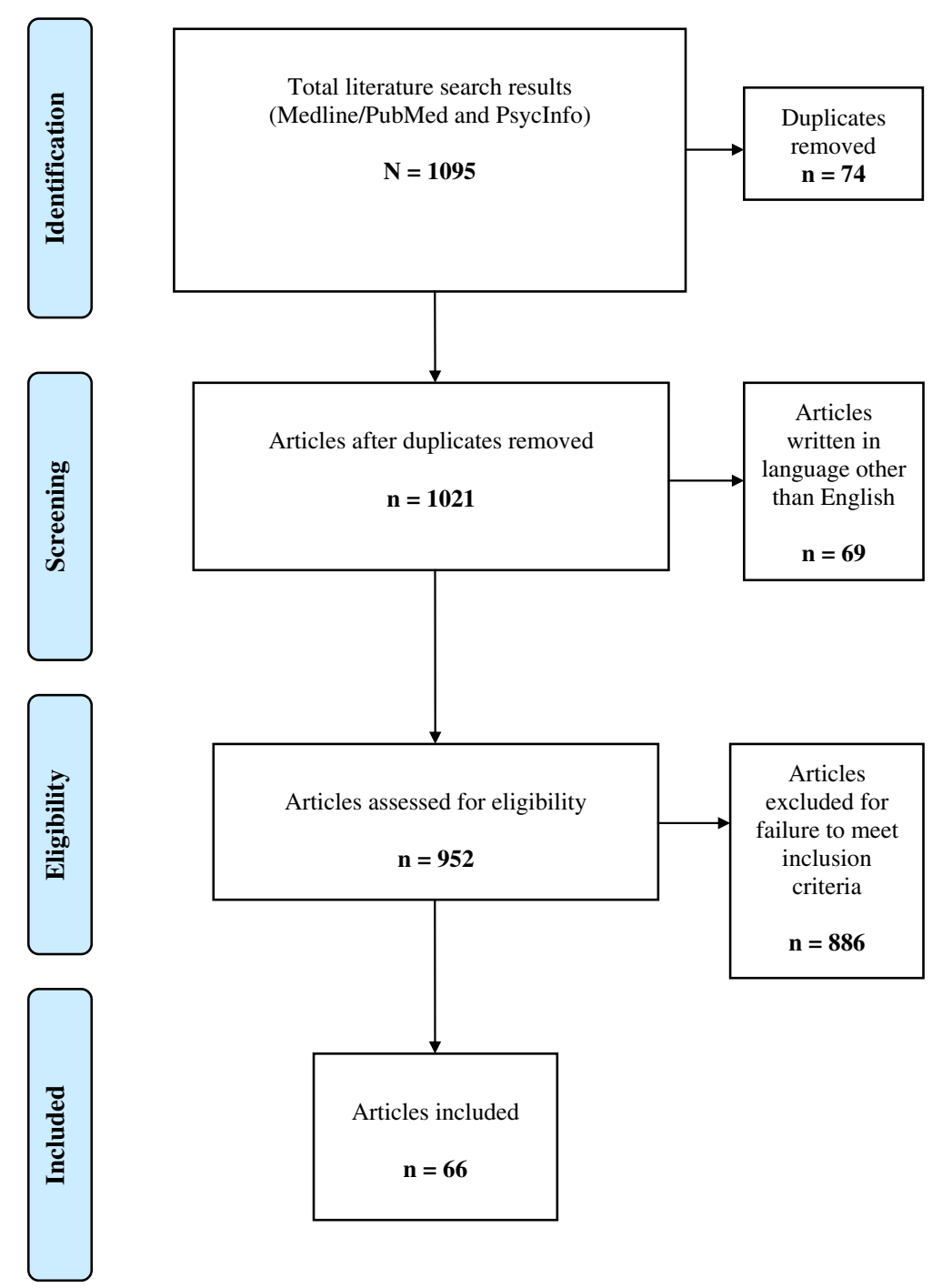

Figure 1 Flow chart of included studies.

suicide deaths in the general population, was 13.6 (95\% CI 10.9-16.8) [45]. The SMR was only exceeded in this sample by deaths due to psychoactive substance use (SMR 18.9, 95\% CI 10.0-32.3) and those caused primarily by AN (SMR 650.0, 95\% CI 462.2-888.6) [45]. In a study that analyzed eight years of follow-up data from 147 outpatients with $\mathrm{AN}$, and combined these data with those from 10 additional studies that included follow-up data from other samples, Signorini et al. reported $5.3 \%$ overall mortality rate, including $1.2 \%$ death due to suicide [56]. In a meta-analysis of 36 studies published between 1966-2010 that included mortality rates in individuals with EDs, Arcelus et al. found a SMR of 5.9 for the AN sample, in which one in five individuals who died had committed suicide [10].

While overall mortality risk, including risk of death by suicide, appears to be significantly higher in AN, compared to risk in individuals with BN and BED [11], there are significant differences in rates of suicidality between AN subtypes: $7.4 \%$ of AN-restricting subjects reported at least one suicide attempt, whereas approximate rates are between 20 and 30\% for other AN subtypes [36]. Selby et al. conducted a two-part cohort study investigating suicidality and associated "provocative behaviors" among AN subtypes including: restricting, purging, binge-purging, and history of $\mathrm{BN}$ [55]. The study found in both cohorts $(n=787$ and replicated $\mathrm{n}=249$ ) that suicidal behavior in AN may be the result of these associated behaviors defined by the AN subtypes. Repetitive experience with behaviors such as binge eating and self-induced vomiting may be a route to suicidal behavior in bingeing and/or purging subtypes, while the exposure to pain through starvation 
Table 1 Papers describing suicide and/or self-injury associated with eating disorders

\begin{tabular}{|c|c|c|c|c|c|}
\hline $\begin{array}{l}\text { Author } \\
N=66\end{array}$ & $\begin{array}{l}\text { Study design } \\
n=70\end{array}$ & $\begin{array}{l}\text { Sample size } \\
n=54\end{array}$ & $\begin{array}{l}\text { ED diagnoses } \\
n=67\end{array}$ & $\begin{array}{l}\text { Age group } \\
n=55\end{array}$ & $\begin{array}{l}\text { Gender } \\
n=52\end{array}$ \\
\hline Ahrén-Moonga, [8] & Clinical cohort with controls & $105^{*}$ & $\mathrm{AN}, \mathrm{BN}$ & Adults & Females \\
\hline Ajetunmobi, [1] & Community cohort & $111504^{*}$ & ED & Adults & Mixed \\
\hline Anestis, [9] & Clinical cohort & 127 & $\mathrm{BN}$ & Adults & Females \\
\hline Arcelus, [10] & Review/meta-analysis & - & AN, BN, EDNOS & - & - \\
\hline Berkman, [11] & Review/meta-analysis & - & $\mathrm{AN}, \mathrm{BN}, \mathrm{BED}$ & - & - \\
\hline Birmingham, [12] & Clinical cohort & 954 & AN, BN, EDNOS & Adults & Females \\
\hline Bodell, [13] & Community cohort & 364 & AN, BN, EDNOS & Adults & Females \\
\hline Bulik, [14] & Clinical cohort & 432 & $\mathrm{AN}, \mathrm{BN}$ & Adolescents and adults & Mixed \\
\hline Button [15] & Clinical cohort & 1892 & $\mathrm{AN}, \mathrm{BN}, \mathrm{EDNOS}$ & Adults & Females \\
\hline Carano, [16] & Clinical cohort & 80 & BED & Adults & Mixed \\
\hline Chen, [17] & Clinical cohort & 135 & $\mathrm{AN}, \mathrm{BN}, \mathrm{BED}$ & Adults & Females \\
\hline Claes, [18] & Clinical cohort & $70^{*}$ & $\mathrm{AN}, \mathrm{BN}$ & Adults & Females \\
\hline Claes, [19] & Clinical cohort & 130 & AN, BN, EDNOS & Adults & Males \\
\hline Crow, [20] & Clinical cohort & 1885 & AN, BN, EDNOS & Adults & - \\
\hline Dancyger, [21] & Review/meta-analysis & - & AN, BN, EDNOS & Adolescents & - \\
\hline Erdur, [22] & Clinical cohort & $169^{*}$ & AN & Adolescents and adults & Females \\
\hline Favaro, [23] & Clinical cohort & 934 & $\mathrm{AN}, \mathrm{BN}, \mathrm{ED}$ & Adults & Females \\
\hline Favaro, [24] & Clinical cohort & 95 & $\mathrm{BN}$ & Adults & - \\
\hline Fedorowicz, [25] & Clinical cohort & $968^{*}$ & AN, BN, EDNOS & Adolescents and adults & Females \\
\hline Fennig, [26] & Clinical cohort & 46 & $\mathrm{AN}, \mathrm{BN}$ & Adolescents & Females \\
\hline Fichter, [27] & Clinical cohort & 103 & AN & Adolescents and adults & Females \\
\hline Fischer, [28] & Clinical cohort & 80 & $\mathrm{BN}$ & Adolescents & Mixed \\
\hline Forcano, [29] & Clinical cohort with controls & 172 & AN & Adults & Females \\
\hline Forcano, [30] & Clinical cohort & 566 & BN, EDNOS & Adults & Females \\
\hline Foulon, [2] & Clinical cohort & 304 & AN & Adolescents and adults & Mixed \\
\hline Franko, [5] & Clinical cohort & 246 & $\mathrm{AN}, \mathrm{BN}$ & Adolescents and adults & Females \\
\hline Franko, [7] & Review/meta-analysis & - & $\mathrm{AN}, \mathrm{BN}$ & - & - \\
\hline Fujimori, [31] & Clinical cohort with controls & 200 & ED & Adults & Females \\
\hline Grucza, [32] & Community cohort & 910 & BED & Adults & Mixed \\
\hline Gueguen, [33] & Clinical cohort & $1009^{*}$ & AN & Adults & Mixed \\
\hline Guillaume, [34] & Clinical cohort with controls & $1563^{*}$ & $\mathrm{AN}, \mathrm{BN}$ & Adults & Mixed \\
\hline Harris, [35] & Review/meta-analysis & - & $\mathrm{AN}, \mathrm{BN}, \mathrm{BED}, \mathrm{EDNOS}$ & - & - \\
\hline Herpertz-Dahlmann, [36] & Review/meta-analysis & - & $\mathrm{AN}, \mathrm{BN}, \mathrm{BED}, \mathrm{EDNOS}$ & - & - \\
\hline Hoek, [37] & Review/meta-analysis & - & $\mathrm{AN}, \mathrm{BN}$ & - & - \\
\hline Huas, [38] & Clinical cohort & $601^{*}$ & AN & Adults & Females \\
\hline Huas, [39] & Clinical cohort with controls & $258^{*}$ & $\mathrm{BN}$ & Adolescents and adults & Females \\
\hline Latzer, [40] & Review/meta-analysis & - & AN & - & - \\
\hline Liang, [41] & Clinical cohort with controls & 316 & $\mathrm{AN}, \mathrm{BN}, \mathrm{EDNOS}$ & Adolescents and adults & Females \\
\hline Millar, [42] & Clinical cohort & 524 & AN & Adolescents and adults & Females \\
\hline Muehlenkamp, [43] & Clinical cohort & $422^{*}$ & $\mathrm{AN}, \mathrm{BN}, \mathrm{BED}, \mathrm{EDNOS}$ & Adults & Females \\
\hline Muehlenkamp, [44] & Clinical cohort & 131 & $\mathrm{BN}$ & Adults & Females \\
\hline Nickel, [3] & Community cohort & 813 & $\mathrm{BN}$ & Adolescents and adults & Females \\
\hline Papadopoulos, [45] & Clinical cohort & $6009^{*}$ & AN & Adolescents and adults & Females \\
\hline
\end{tabular}


Table 1 Papers describing suicide and/or self-injury associated with eating disorders (Continued)

\begin{tabular}{|c|c|c|c|c|c|}
\hline Papadopoulos, [46] & Clinical cohort & 5251 & AN & Adolescents and adults & Females \\
\hline Peebles, [47] & Clinical cohort & 1432 & ED & Adolescents and adults & Mixed \\
\hline Peterson, [48] & Clinical cohort & 489 & BN & Adolescents and adults & Females \\
\hline Pisetsky, [4] & Clinical cohort & 13035 & $A N, B N, B E D$ & Adults & Females \\
\hline Pompili, [49] & Review/meta-analysis & - & AN, BN & - & - \\
\hline Preti, [50] & Review/meta-analysis & - & $A N, B N, B E D$ & - & - \\
\hline Rigaud, [51] & Clinical cohort & $484^{*}$ & AN & Adolescents and adults & Mixed \\
\hline Rosling, [52] & Clinical cohort & $201^{*}$ & AN, BN & Adolescents and adults & Mixed \\
\hline Ross, [6] & Community cohort with controls & 440 & ED & Adolescents & Mixed \\
\hline Ruuska, [53] & Clinical cohort & 57 & $A N, B N$ & Adolescents & Females \\
\hline Schroeder, [54] & Clinical cohort with controls & 69 & $\mathrm{AN}, \mathrm{BN}$ & Adults & Females \\
\hline \multirow[t]{2}{*}{ Selby, [55] } & Clinical cohort & 787 & AN & Adolescents and adults & Mixed \\
\hline & Clinical cohort & 249 & AN & Adolescents and adults & Mixed \\
\hline Signorini, [56] & Clinical cohort & 2240 & AN & Adults & Females \\
\hline Silber, [57] & Review/meta-analysis & - & $A N, B N$ & - & - \\
\hline Smink, [58] & Review/meta-analysis & - & $\mathrm{AN}, \mathrm{BN}, \mathrm{BED}, \mathrm{EDNOS}$ & - & - \\
\hline Smink, [59] & Review/meta-analysis & - & AN, BN, BED, EDNOS & - & - \\
\hline \multirow[t]{4}{*}{ Smith, [60] } & Clinical cohort & 204 & $\mathrm{BN}$ & Adolescents and adults & Females \\
\hline & Community cohort & 171 & - & Adolescents and adults & Mixed \\
\hline & Community cohort & 467 & - & Adolescents and adults & Mixed \\
\hline & Community cohort & 512 & - & Adolescents and adults & Mixed \\
\hline Steinhausen, [61] & Review/meta-analysis & - & $A N, B N$ & - & - \\
\hline Steinhausen, [62] & Review/meta-analysis & - & $\mathrm{BN}$ & - & - \\
\hline Suokas, [63] & Clinical cohort with controls & 2450 & AN, BN, BED & adults & Mixed \\
\hline Svirko, [64] & Review/meta-analysis & - & AN, BN, EDNOS & - & - \\
\hline Tozzi, [65] & Clinical cohort & 1021 & $\mathrm{AN}, \mathrm{BN}, \mathrm{EDNOS}$ & Adolescents and adults & Mixed \\
\hline Vansteelandt, [66] & Clinical cohort & 59 & $\mathrm{AN}, \mathrm{BN}$ & Adults & Females \\
\hline
\end{tabular}

Note. All ED diagnoses are based on DSM-IV. The following abbreviations are used: $\mathrm{AN}=$ anorexia nervosa; $\mathrm{BN}=$ bulimia nervosa;

$\mathrm{BED}=$ binge eating disorder; $\mathrm{ED}=$ eating disorders, general; $\mathrm{EDNOS}=$ eating disorder not otherwise specified.

*inpatient.

from restriction may lead to suicidal behavior in restricting subtypes $[29,51,55]$.

Other factors that have been specifically examined in relation to suicidality in populations with $\mathrm{AN}$ include patients' age at assessment, pregnancy and gender. Arcelus et al. found that the age of the patient at the time of ED assessment is a significant predictor of mortality, including death by suicide [10]. Pregnancy has also been examined in relation to suicidality and AN. In a population-based analysis, 5251 females with AN were evaluated, comparing suicide rates between a parous and a nulliparous group [46]. Suicide was the most common cause of death in both groups. The SMR for suicide in the parous group was 6.3 (95\% CI 2.7-12.3) compared to 16.1 in the nulliparous group (95\% CI 1.8-21.4), and in fact, childbearing was associated with $65 \%$ lower general mortality in AN [46]. While few studies evaluate AN in the male population due to the small sample sizes,
Gueguen et al. conducted a longitudinal cohort study comparing 23 men with AN to 601 women with AN and found that men with AN were less likely to have a history of a suicide attempts compared to women with AN (4\% vs. $29 \%$; $=0.01$ ) [33].

\section{Bulimia nervosa}

Previously, data have shown that mortality rates in general are lower in populations with a diagnosis of $\mathrm{BN}$ than in populations with a diagnosis of $\mathrm{AN}$, but few data have specified rates of suicidality in $\mathrm{BN}$. For example, the meta-analysis from Arcelus et al. and a longitudinal study from Franko et al. both examined the mortality rates for $\mathrm{BN}$ : Arcelus et al. found a weighted mortality rate of 1.7 (95\% CI 1.09-2.44) per 1000 person-years, compared to a weighted mortality rate of 5.1 for $\mathrm{AN}$ [10], and Franko's findings were similar [5], but neither of these reports mentioned suicide as a cause of death in 
the BN population. One study that did look at suicide in $\mathrm{BN}$ found that characteristics of suicide attempts did not significantly differ between $\mathrm{BN}$ and controls with no ED diagnosis [34].

With regard to completed suicides in $\mathrm{BN}$, findings are mixed. Rates of suicide in both the BN and control samples from Guillaume et al. were altogether lower than in patients with AN [34]. A meta-analysis by Preti et al. reported that in BN, suicide as the cause of death was present in roughly the same proportion as that seen in AN [50]. Crow and colleagues reported a larger percentage of death due to suicide in a BN sample $(0.9 \%)$ versus an AN sample (0.6\%) [20]. Overall, however, the literature indicates that the number of completed suicides among BN appears to be low in comparison to AN.

Some studies have focused on suicidal ideation and suicide attempts, as opposed to completed suicides, and have found that, consistent with the earlier findings of Franko and Keel, suicide attempts are more prevalent in $\mathrm{BN}$ populations than in AN populations [7]. Investigating the risk for suicidality between patients with $\mathrm{AN}, \mathrm{BN}$ and EDNOS, Bodell and colleagues found that BN was significantly associated with suicidality independent of risk predicted by comorbid disorders, while neither AN nor EDNOS were found to be uniquely associated with suicidality [13]. In a meta-analysis by Fedorowicz and colleagues, BN was most strongly associated with suicide attempts or suicidal ideation, followed by AN-binge/ purge subtype [25]. Ahrén-Moonga and colleagues investigated the prevalence of suicide plans and/or thoughts, as well as history of suicide attempts in inpatients with EDs, and found a higher prevalence of suicide attempts associated with BN compared with AN $(\mathrm{p}<0.02)$ [8]. Forcano et al. found a lifetime prevalence of suicide attempts to be $26.9 \%(\mathrm{n}=152,95 \%$ CI 23.2 30.5) in a sample of $566 \mathrm{BN}$ outpatients [30]. More recently published data from Smith et al. found that approximately one-third of women with a diagnosis of $\mathrm{BN}$ have had at least one suicide attempt [60]. There are fewer published data on adolescents with $\mathrm{BN}$ than on adults, but a diagnosis of $\mathrm{BN}$ in adolescents does appear to raise the risk of increased suicidality and self-harm. For example, in a study that looked at 80 adolescents with BN, $25 \%$ reported having attempted suicide or engaging in self-injurious behavior [28]. A study comparing adolescents with $\mathrm{AN}$ to adolescents with $\mathrm{BN}$ found that the BN group had significantly more suicidal ideation than the AN group [53].

\section{Binge eating disorder and other categories of eating disorders}

Less is known about the prevalence of suicidality in populations with a specific diagnosis of BED, or in individuals previously diagnosed with EDNOS according to
DSM-IV, or other categories of illness described in the DSM-5 such as ARFID. BED was described in DSM-IV but identified as a formal diagnostic category only recently with the publication of DSM-5. The authors identified few studies that specifically examine suicide associated with BED. Among collected data from community samples, subjects with BED have shown elevated odds for suicide attempts $[4,32]$. In a thorough examination of all deaths reported among a clinical cohort of 2450 individuals with EDs and a control population, followed for up to 15 years (average length of follow-up was 8.7 years), Suokas et al. identified that four deaths occurred among the 171 individuals with broadly defined BED, one of which was due to suicide [63]. Pisetsky et al. reported that $19 \%$ of a sample that met criteria for a narrow diagnosis of BED, and 59\% of a sample that met for a broad diagnosis of BED, had a lifetime prevalence of at least one suicide attempt [4]. No results were produced from literature searches using ED key words "avoidant/restrictive food intake disorder" or the acronym "ARFID".

\section{Other risk factors of suicidality in eating disorders}

The literature described by Franko and Keel in their 2006 review as well as more recent publications identified in this review consistently emphasize the clinical importance of identifiable risk factors associated with suicidality in EDs. Individual studies have identified a range of factors that may be associated with increased risk of suicidality and/or associated behaviors among individuals with EDs. Illness severity [26], co-occurring psychiatric illnesses including borderline personality disorder [17] and substance abuse [25], excessive exercise [60], and alexithymia [16] have been mentioned as possible risk factors. With regard to age groups, Dancyger and Fomari found that across ED diagnoses, adolescents demonstrate a strong relationship between suicidal behavior and completed suicides [21]. Dancyger and Fomari's review concluded with an emphasis on the importance of clinical awareness to the heightened risk of suicide in the adolescent ED population, the treatment for which may present to be challenging as it requires careful coordination between clinicians, parents, and the young adult patients themselves.

\section{Self-injurious behaviors}

In addition, data from reviews, clinical longitudinal investigations, and community cohort studies, show significant associations between EDs and self-injurious behaviors $[18,23,60,64,65]$. Self-injurious behaviors, which may include compulsive behaviors (e.g., hair pulling, nail biting, skin picking, self-biting) and impulsive self-injurious behaviors (e.g., cutting, burning, self-hitting, banging, scratching), are sometimes referred to as NSSI because the individuals 
engaging in the behaviors are doing so with the intention of physical self-harm, but without suicidal intent [23]. However, these behaviors, considered on a spectrum of self-destructive behaviors, may be associated with risk of suicidality $[23,65]$.

Data on the relationship between self-injury and EDs indicate that self-injury has a higher prevalence in ED patients with a history of $\mathrm{BN}$, binge eating, or purging behaviors. Tozzi et al. focused specifically on laxative abuse and found in samples of AN and BN that the function of laxative abuse included both a method of purging as well as a form of selfharm [65]. In a case study of 70 female inpatients with EDs, Claes et al. found that $38.6 \%$ engaged in NSSI [18]. Of the NSSI and ED group, a significantly higher portion of individuals with BN engaged in NSSI than did individuals with AN $(\mathrm{p}<0.05)$ [18]. Ahrén-Moonga and colleagues found that $36.8 \%$ of a group of $38 \mathrm{ED}$ patients engaged in self-injury [8]. Fourteen of the patients also reported suicidal ideation or plans, and 10 patients had a history of suicide attempts. Consistent with other studies $[18,23,60,64,65]$, these data show a higher prevalence of self-injury-as well as suicide attempts-in BN compared with $\mathrm{AN}(\mathrm{p}<0.05$ and 0.02 , respectively) [8].

Other factors that appear to positively influence the relationship between EDs and self-injury include depression, impulsivity, obsessive-compulsiveness, affect dysregulation, dissociation, self-criticizing cognitive style, and need for control, as well as affective lability and previous suicidal behavior or attempts $[9,53,64]$. Consistent with previous reports, the current review found support for a strong association between EDs and self-injury among individuals with EDs who report a history of trauma and abuse, including verbal, emotional, neglectful, physical, sexual, and/or substance abuse $[8,18,24,43,47]$. Furthermore, one study that looked specifically at the relationship between pregnancy and self-injury in an ED sample found that, while the majority of women with EDs experience improved ED symptoms during pregnancy, some women in this sample, particularly those diagnosed with binge eating pathology or subclinical ED diagnoses, had a risk of experiencing escalated pathological behaviors during pregnancy [35].

These additional risk factors that may co-occur with or even result in self-injury seem to correlate with more severe ED pathology among ED individuals. In a longitudinal follow-up study, Peterson et al. found that young-adult BN patients who engaged in NSSI at baseline showed an association with increased purging at follow-up eight months after initial presentation [48]. The results also showed that negative urgency at baseline was a significant predictor of NSSI and BN symptoms at follow-up. Fujimori and colleagues found that among individuals with EDs, those who reported self-injury had higher Eating Disorder Inventory scores, signifying greater severity of ED symptoms, than those without self-injury $(p<0.001)$ [31]. In Muehlenkamp and colleagues' study of 422 inpatient ED females, 34.6\% of participants engaged in NSSI [43]. This investigation revealed that no specific ED diagnosis was associated with self-injury more commonly than another, but the ED types that specifically include binge or purge behaviors (e.g. AN-BP and $\mathrm{BN}$ ) were associated with a significantly higher number of types of NSSI including cutting, scratching, bruising, and burning, compared to individuals with other ED types $\left(\mathrm{X}^{2}(16)=\right.$ 32.94, $\mathrm{P}<0.01)$ [43]. In an all-male ED sample $(\mathrm{N}=130)$, Claes and colleagues found that $20.8 \%$ reported engaging in NSSI [19]. The NSSI group showed significantly more ED symptoms and had an overall more severe presentation than the non-self-injurious ED males. While there was no significant difference in the rate of NSSI occurring among the three ED diagnostic groups in the sample (AN, BN, and EDNOS), there was a tendency for a higher rate of NSSI among the BN group [19].

Self-injurious behaviors are especially common among adolescents with ED symptoms. Even among adolescents without specific ED diagnoses, results indicate that students who engage in NSSI display significantly more eating pathology, including increased body dissatisfaction and bulimic tendencies (especially binge eating), than their non-NSSI peers [6]. Peebles et al. conducted a chart review of 1432 adolescent ED patients over a nineyear period and found that $40.8 \%$ of the sample reported engaging in self-injury [47]. Of those ED adolescents reporting self-injury, females were more likely than males to engage in self-harm behaviors, the most common of which was cutting (85.2\%). In this sample, there was also a strong association between self-injury and history of abuse $(\mathrm{p}<0.05)[47]$.

\section{Conclusion}

As a group, despite the variation in the content of the existing literature, the 66 publications described in this narrative review support the finding of a consistently high prevalence of suicidality associated with EDs. Specifically, AN is consistently associated with high rates of suicide, especially in the context of significantly elevated SMRs for adolescents and young adults. Contrary to previous findings, some recent studies have found that suicide risk appears elevated in $\mathrm{BN}$ as well, although there is more variability in the literature on BN study findings; this may be due to sample differences across studies, including the possibility that some individuals with BN may have a history of AN. Self-injurious behaviors are frequent in EDs, with higher rates among EDs that include binge and purge behaviors. Self-injurious behaviors, which may or may not include suicidal intent, are especially high among adolescents with EDs. Furthermore, recent investigations on individuals who engage in rigorous physical exercise are important because they suggest that overexercise - often considered a self-injurious behavior among ED individuals - may pose additional risk beyond the 
physical consequence of the activity itself. Over-exercise may change pain sensation and in turn affect individuals' acquired capability for suicide. Based on these findings, individuals with EDs, including those with BN symptoms, who engage in over-exercise, may be at higher risk for suicide. These data underscore the importance of assessing for suicide risk not just in individuals with $\mathrm{AN}$, where suicide rates have long been recognized as elevated, but also in individuals presenting with $\mathrm{BN}$ and other ED symptoms. Ultimately, there are a number of risk factors associated with suicidality, which may occur across ED diagnoses. Clinicians must pay close attention to these factors, including chronicity of illness, comorbid diagnoses, history of abuse, and self-injurious behaviors, as occurrence of these factors may increase the potential risk of suicidality.

\section{Limitations}

There are several limitations with regard to this review's assessment of the prevalence of suicidality and self-injury in EDs. Methodologically, the review is narrative without a quantitative aggregation of data. While all authors read the reviews that resulted in the literature search, the reviewing of only papers in English limited the quantity of literature search results reviewed. Additionally, the authors' search for the DSM-5's newly described diagnosis ARFID produced no results. Perhaps future published data will include the prevalence of suicidality in EDs, including ARFID, corresponding to newly described criteria in the DSM-5. Ultimately, while more standardized investigation is necessary, multiple studies have clearly found a high risk of mortality, including risk of suicide and self-injurious behaviors, among patients with EDs.

\section{Abbreviations \\ AN: Anorexia nervosa; ARFID: Avoidant/restrictive food intake disorder; BED: Binge eating disorder; BN: Bulimia nervosa; Cl: Confidence interval; ED: Eating disorder; EDNOS: Eating disorder not otherwise specified; NSSI: Non-suicidal self-injury; OR: Odds ratio; SMR: Standardized mortality ratio.}

\section{Competing interests}

The authors declare that they have no competing interests. Dr. Attia receives research support from Eli Lilly and Co. Publication of this manuscript was supported by Beyondblue.

\section{Authors' contributions}

All authors carried out the literature search, contributed to the development of this review, and drafted the manuscript. All authors read and approved the final manuscript.

\section{Author details}

'Columbia University Medical Center, New York, NY, USA. ${ }^{2}$ Weill Cornell Medical College, New York, NY, USA.

Received: 6 December 2013 Accepted: 23 June 2014

Published: 7 July 2014

\section{References}

1. Ajetunmobi O, Taylor M, Stockton D, Wood R: Early death in those previously hospitalised for mental healthcare in Scotland: a nationwide cohort study, 1986-2010. BMJ Open 2013, 3(7):1-9.
2. Foulon C, Guelfi JD, Kipman A, Adès J, Romo L, Houdeyer K, Marquez S, Mouren MC, Rouillon F, Gorwood P: Switching to the bingeing/purging subtype of anorexia nervosa is frequently associated with suicidal attempts. Eur Psychiatry 2007, 8:513-519.

3. Nickel MK, Simek M, Lojewski N, Muehlbacher M, Fartacek R, Kettler C, Bachler E, Egger C, Rother N, Buschmann W, Pedrosa Gil F, Kaplan P, Mitterlehner FO, Anvar J, Rother WK, Loew TH, Nickel C: Familial and sociopsychopathological risk factors for suicide attempt in bulimic and in depressed women: prospective study. Int J Eat Disord 2006, 39(5):410-417.

4. Pisetsky EM, Thornton LM, Lichtenstein P, Pedersen NL, Bulik CM: Suicide attempts in women with eating disorders. J Abnorm Psychol 2013, 122(4):1042-1056.

5. Franko DL, Keshaviah A, Eddy KT, Krishna M, Davis MC, Keel PK, Herzog DB: A longitudinal investigation of mortality in anorexia nervosa and bulimia nervosa. Am J Psychiatry 2013, 170(8):917-925.

6. Ross S, Heath NL, Toste JR: Non-suicidal self-injury and eating pathology in high school students. Am J Orthopsychiatry 2009, 79(1):83-92.

7. Franko DL, Keel PK: Suicidality in eating disorders: occurrence, correlates, and clinical implications. Clin Psychol Rev 2006, 26(6):769-782.

8. Ahrén-Moonga J, Holmgren S, von Knorring L, Af Klinteberg B: Personality traits and self-injurious behaviour in patients with eating disorders. Eur Eat Disord Rev 2008, 16(4):268-275.

9. Anestis MD, Silva C, Lavender JM, Crosby RD, Wonderlich SA, Engel SG, Joiner TE: Predicting nonsuicidal self-injury episodes over a discrete period of time in a sample of women diagnosed with bulimia nervosa: an analysis of self-reported trait and ecological momentary assessment based affective lability and previous suicide attempts. Int J Eat Disord 2012, 45(6):808-811.

10. Arcelus J, Mitchell AJ, Wales J, Nielsen S: Mortality rates in patients with anorexia nervosa and other eating disorders: a meta-analysis of 36 studies. Arch Gen Psychiatry 2011, 68(7):724-731.

11. Berkman ND, Lohr KN, Bulik CM: Outcomes of eating disorders: a systematic review of the literature. Int J Eat Disord 2007, 40(4):293-309.

12. Birmingham CL, Su J, Hlynsky JA, Goldner EM, Gao M: The mortality rate from anorexia nervosa. Int J Eat Disord 2005, 38(2):143-146.

13. Bodell LP, Joiner TE, Keel PK: Comorbidity-independent risk for suicidality increases with bulimia nervosa but not with anorexia nervosa. J Psychiatr Res 2013, 47(5):617-621.

14. Bulik CM, Thornton L, Pinheiro AP, Plotnicov K, Klump KL, Brandt H, Crawford S, Fichter MM, Halmi KA, Johnson C, Kaplan AS, Mitchell J, Nutzinger D, Strober M, Treasure J, Woodside DB, Berrettini WH, Kaye WH: Suicide attempts in anorexia nervosa. Psychosom Med 2008, 70(3):378-383.

15. Button E, Chadalavada B, Palmer RL: Mortality and predictors of death in a cohort of patients presenting to an eating disorders service. Int J Eat Disord 2010, 43(5):387-392

16. Carano A, De Berardis D, Campanella D, Serroni N, Ferri F, Di lorio G, Acciavatti T, Mancini L, Mariani G, Martinotti G, Moschetta FS, Di Giannantonio M: Alexithymia and suicide ideation in a sample of patients with binge eating disorder. J Psychiatr Pract 2012, 18(1):5-11.

17. Chen EY, Brown MZ, Harned MS, Linehan MM: A comparison of borderline personality disorder with and without eating disorders. Psychiatry Res 2009, 170(1):86-90

18. Claes L, Vandereycken $\mathrm{W}$ : Is there a link between traumatic expriences and self-injurious behaviors in eating-disordered patients? Eat Disord 2007, 15(4):305-315.

19. Claes L, Jimenez-Murcia S, Aguera Z, Castro R, Sanchez I, Manuel Menchon J, Fernandez-Aranda F: Male eating disorder patients with and without non-suicidal self-injury: A comparison of psychopathological and personality features. Eur Eat Disord Rev 2012, 20(4):335-338.

20. Crow SJ, Peterson CB, Swanson SA, Raymond NC, Specker S, Eckert ED, Mitchell JE: Increased mortality in bulimia nervosa and other eating disorders. Am J Psychiatry 2009, 166(12):1342-1346.

21. Dancyger IF, Fornari VM: A review of eating disorders and suicide risk in adolescence. ScientificWorldJournal 2005, 5:803-811.

22. Erdur L, Kallenbach-Dermutz B, Lehmann V, Zimmermann-Viehoff F, Köpp W, Weber C, Deter HC: Somatic comorbidity in anorexia nervosa: First results of a 21-year follow-up study on female inpatients. Biopsychosoc Med 2012, 6(1):4.

23. Favaro A, Ferrara S, Santonastaso P: Self-injurious behavior in a community sample of young women: relationship with childhood abuse and other types of self-damaging behaviors. J Clin Psychiatry 2007, 68(1):122-131. 
24. Favaro A, Santonastaso P, Monteleone P, Bellodi L, Mauri M, Rotondo A, Erzegovesi S, Maj M: Self-injurious behavior and attempted suicide in purging bulimia nervosa: associations with psychiatric comorbidity. $J$ Affect Disord 2008, 105(1-3):285-289.

25. Fedorowicz VJ, Falissard B, Foulon C, Dardennes R, Divac SM, Guelfi JD, Rouillon F: Factors associated with suicidal behaviors in a large French sample of inpatients with eating disorders. Int J Eat Disord 2007 40(7):589-595.

26. Fennig $S$, Hadas A: Suicidal behavior and depression in adolescents with eating disorders. Nord J Psychiatry 2010, 64(1):32-39.

27. Fichter MM, Quadflieg N, Hedlund S: Twelve-year course and outcome predictors of anorexia nervosa. Int J Eat Disord 2006, 39(2):87-100.

28. Fischer $\mathrm{S}$, le Grange D: Comorbidity and high-risk behaviors in treatmentseeking adolescents with bulimia nervosa. Int J Eat Disord 2007, 40(8):751-753.

29. Forcano L, Alvarez E, Santamaría JJ, Jimenez-Murcia S, Granero R, Penelo E, Alonso P, Sánchez I, Menchón JM, Ulman F, Bulik CM, Fernández-Aranda F: Suicide attempts in anorexia nervosa subtypes. Compr Psychiatry 2011 52(4):352-358

30. Forcano L, Fernández-Aranda F, Alvarez-Moya E, Bulik C, Granero R, Gratacòs M, Jiménez-Murcia S, Krug I, Mercader JM, Riesco N, Saus E, Santamaría JJ, Estivill X: Suicide attempts in bulimia nervosa: personality and psychopathological correlates. Eur Psychiatry 2009, 24(2):91-97.

31. Fujimori A, Wada Y, Yamashita T, Choi H, Nishizawa S, Yamamoto H, Fukui K: Parental bonding in patients with eating disorders and self-injurious behavior. Psychiatry Clin Neurosci 2011, 65(3):272-279.

32. Grucza RA, Przybeck TR, Cloninger CR: Prevalence and correlates of binge eating disorder in a community sample. Compr Psychiatry 2007, 48(2):124-131.

33. Gueguen J, Godart N, Chambry J, Brun-Eberentz A, Foulon C, Divac Phd SM, Guelfi JD, Rouillon F, Falissard B, Huas C: Severe anorexia nervosa in men: comparison with severe AN in women and analysis of mortality. Int J Eat Disord 2012, 45(4):537-545.

34. Guillaume S, Jaussent I, Olié E, Genty C, Bringer J, Courtet P, Schmidt U: Characteristics of suicide attempts in anorexia and bulimia nervosa: a case-control study. PLoS One 2011, 6(8):e23578.

35. Harris AA: Practical advice for caring for women with eating disorders during the perinatal period. J Midwifery Womens Health 2010, 55(6):579-586.

36. Herpertz-Dahlmann B: Adolescent eating disorders: definitions, symptomatology, epidemiology and comorbidity. Child Adolesc Psychiatr Clin N Am 2009, 18(1):31-47.

37. Hoek HW: Incidence, prevalence and mortality of anorexia nervosa and other eating disorders. Curr Opin Psychiatry 2006, 19(4):389-394.

38. Huas C, Caille A, Godart N, Foulon C, Pham-Scottez A, Divac S, Dechartres A, Lavoisy G, Guelfi JD, Rouillon F, Falissard B: Factors predictive of ten-year mortality in severe anorexia nervosa patients. Acta Psychiatr Scand 2011, 123(1):62-70.

39. Huas C, Godart N, Caille A, Pham-Scottez A, Foulon C, Divac SM, Lavoisy G, Guelfi JD, Falissard B, Rouillon F: Mortality and its predictors in severe bulimia nervosa patients. Eur Eat Disord Rev 2013, 21(1):15-19.

40. Latzer $Y$, Hochdorf Z: Dying to be thin: attachment to death in anorexia nervosa. ScientificWorldJournal 2005, 5:820-827.

41. Liang KY, Meg Tseng MC: Impulsive behaviors in female patients with eating disorders in a university hospital in northern Taiwan. J Formos Med Assoc 2011, 110(9):607-610.

42. Millar HR, Wardell F, Vyvyan JP, Naji SA, Prescott GJ, Eagles JM: Anorexia nervosa mortality in Northeast Scotland, 1965-1999. Am J Psychiatry 2005, 162(4):753-757.

43. Muehlenkamp JJ, Claes L, Smits D, Peat CM, Vandereycken W: Non-suicidal self-injury in eating disordered patients: a test of a conceptual model. Psychiatry Res 2011, 188(1):102-108.

44. Muehlenkamp JJ, Engel SG, Wadeson A, Crosby RD, Wonderlich SA, Simonich $H$, Mitchell JE: Emotional states preceding and following acts of non-suicidal self-injury in bulimia nervosa patients. Behav Res Ther 2008, 47(1):83-87.

45. Papadopoulos FC, Ekbom A, Brandt L, Ekselius L: Excess mortality, causes of death and prognostic factors in anorexia nervosa. Br J Psychiatry 2009, 194(1):10-17.

46. Papadopoulos FC, Karamanis G, Brandt L, Ekbom A, Ekselius L: Childbearing and mortality among women with anorexia nervosa. Int J Eat Disord 2013, 46(2):164-170
47. Peebles R, Wilson JL, Lock JD: Self-injury in adolescents with eating disorders: correlates and provider bias. J Adolesc Health 2011, 48(3):310-313

48. Peterson CM, Fischer S: A prospective study of the influence of the UPPS model of impulsivity on the co-occurrence of bulimic symptoms and non-suicidal self-injury. Eat Behav 2012, 13(4):335-341.

49. Pompili M, Girardi P, Tatarelli G, Ruberto A, Tatarelli R: Suicide and attempted suicide in eating disorders, obesity and weight-image concern. Eat Behav 2006, 7(4):384-394.

50. Preti A, Rocchi MB, Sisti D, Camboni MV, Miotto P: A comprehensive meta-analysis of the risk of suicide in eating disorders. Acta Psychiatr Scand 2011, 124(1):6-17.

51. Rigaud D, Tallonneau I, Brindisi MC, Vergès B: Prognosis in 41 severely malnourished anorexia nervosa patients. Clin Nutr 2012, 31(5):693-698.

52. Rosling AM, Sparén P, Norring C, von Knorring AL: Mortality of eating disorders: a follow-up study of treatment in a specialist unit 1974-2000. Int J Eat Disord 2011, 44(4):304-310.

53. Ruuska J, Kaltiala-Heino R, Rantanen P, Koivisto AM: Psychopathological distress predicts suicidal ideation and self-harm in adolescent eating disorder outpatients. Eur Child Adolesc Psychiatry 2005, 14(5):276-281.

54. Schroeder M, Eberlein C, de Zwaan M, Kornhuber J, Bleich S, Frieling H: Lower levels of cannabinoid 1 receptor mRNA in female eating disorder patients: association with wrist cutting as impulsive self-injurious behavior. Psychoneuroendocrinology 2012, 37(12):2032-2036.

55. Selby EA, Smith AR, Bulik CM, Olmsted MP, Thornton L, McFarlane TL, Berrettini WH, Brandt HA, Crawford S, Fichter MM, Halmi KA, Jacoby GE, Johnson CL, Jones I, Kaplan AS, Mitchell JE, Nutzinger DO, Strober M, Treasure J, Woodside DB, Kaye WH, Joiner TE Jr: Habitual starvation and provocative behaviors: two potential routes to extreme suicidal behavior in anorexia nervosa. Behav Res Ther 2010, 48(7):634-645.

56. Signorini A, De Filippo E, Panico S, De Caprio C, Pasanisi F, Contaldo F: Long-term mortality in anorexia nervosa: a report after an 8-year followup and a review of the most recent literature. Eur J Clin Nutr 2007, 61(1):119-222

57. Silber TJ: Ipecac syrup abuse, morbidity, and mortality: isn't it time to repeal its over-the counter status? J Adolesc Health 2005, 37(3):256-260.

58. Smink FR, van Hoeken D, Hoek HW: Epidemiology, course, and outcome of eating disorders. Curr Opin Psychiatry 2013, 26(6):543-548.

59. Smink FR, van Hoeken D, Hoek HW: Epidemiology of eating disorders: incidence, prevalence and mortality rates. Curr Psychiatry Rep 2012 14(4):406-414

60. Smith AR, Fink EL, Anestis MD, Ribeiro JD, Gordon KH, Davis H, Keel PK, Bardone-Cone AM, Peterson CB, Klein MH, Crow S, Mitchell JE, Crosby RD, Wonderlich SA, le Grange D, Joiner TE Jr: Exercise caution: over-exercise is associated with suicidality among individuals with disordered eating. Psychiatry Res 2013, 206(2-3):246-255.

61. Steinhausen HC: Outcome of eating disorders. Child Adolesc Psychiatr Clin N Am 2009, 18(1):225-242

62. Steinhausen HC, Weber S: The outcome of bulimia nervosa: findings from one-quarter century of research. Am J Psychiatry 2009, 166(12):1331-1341.

63. Suokas JT, Suvisaari JM, Gissler M, Löfman R, Linna MS, Raevuori A, Haukka J: Mortality in eating disorders: A follow-up study of adult eating disorder patients treated in tertiary care, 1995-2010. Psychiatry Res 2013, 210(3):1101-1106.

64. Svirko $E_{1}$ Hawton K: Self-injurious behavior and eating disorders: the extent and nature of the association. Suicide Life Threat Behav 2007, 37(4):409-421.

65. Tozzi F, Thornton LM, Mitchell J, Fichter MM, Klump KL, Lilenfeld LR, Reba L, Strober M, Kaye WH, Bulik CM, Price Foundation Collaborative Group: Features associated with laxative abuse in individuals with eating disorders. Psychosom Med 2006, 68(3):470-477.

66. Vansteelandt K, Claes L, Muehlenkamp J, De Cuyper K, Lemmens J, Probst M, Vanderlinden J, Pieters G: Variability in affective activation predicts non-suicidal self-injury in eating disorders. Eur Eat Disord Rev 2013, 21(2):143-147

\section{doi:10.1186/s40337-014-0019-x}

Cite this article as: Kostro et al:: The current status of suicide and selfinjury in eating disorders: a narrative review. Journal of Eating Disorders 2014 2:19. 